\title{
Using Stable Isotopes to Probe the Evolution of Planetary Interiors
}

\author{
ANAT SHAHAR, PENG NI AND NICOLE NIE
}

Earth and Planets Laboratory, Carnegie Institution for Science

Presenting Author: ashahar@carnegiescience.edu

The stable isotopes of heavy elements can be used to trace the accretion, differentiation, and evolution of terrestrial planets. A wide range of non-traditional stable isotope systems have been measured in materials from our Solar System. The combination of experiments, theory, and analysis of natural samples is particularly powerful in understanding the isotopic ratios measured. There are many processes which will fractionate stable isotopes throughout the evolution of a planet so it is imperative to consider all possible mechanisms including: core formation, evaporation/condensation, melting/crystallization, as well as low temperature processes on the surface. In this presentation, we will focus on iron isotopes and their evolution throughout the several billion-year history of a planet. Iron is a ubiquitous element in terrestrial and extra-terrestrial settings as well as an element with multiple oxidation states, making it particularly interesting to trace processes occurring at varying conditions during a planet's evolution.

Here we present the results of several recent studies aimed at understanding the processes that have changed the iron isotopic ratio of the silicate mantle of the Earth. Pyrolitic samples formed by a laser levitation furnace at varying oxygen fugacity conditions, have been probed for their force constants by nuclear resonant inelastic $\mathrm{x}$-ray scattering (NRIXS) at the Advanced Photon Source (APS). Further, natural mantle minerals have also been analyzed by NRIXS to investigate their equilibrium fractionation factors. And lastly, metallic inclusions in superdeep diamonds have been analyzed for their iron isotopic ratios. Our results show that in order to uncover the reason for stable isotope ratios found in nature, we need to consider a multitude of processes throughout the history of the sample. By combining older experimental and natural data, as well as these new data, we will discuss how iron isotopes can be used to infer processes occurring on planets and planetary interiors. 